\title{
Simultaneous Multi-Region Background Subtraction for EELS Spectra
}

Jacob T. Held ${ }^{1 *}$, Hwanhui Yun ${ }^{1}$ and K. Andre Mkhoyan ${ }^{1}$

${ }^{1 .}$ Department of Chemical Engineering \& Materials Science, University of Minnesota, Minneapolis, MN, USA.

* Corresponding author: heldx123@umn.edu

The crucial first step in the analysis of electron energy-loss (EEL) spectra is the removal of the background under the ionization edge of interest. It is common practice to fit a $10-30 \mathrm{eV}$ pre-edge window with a single power law of the form $I(E)=A E^{-B}$, where $A$ is the scaling coefficient and $B$ defines the curvature of the background [1]. The fit curve is then extrapolated and subtracted from the spectrum, leaving background-free core-loss features. This approach generally works well for clean core-loss edges above $\sim 100 \mathrm{eV}$ which do not overlap with other signals.

However, in many practical cases, the backgrounds of EEL spectra are less well-behaved due to a combination of noise, overlap with nearby edges, and/or bulk plasmon contributions to lower energy-loss edges and thick samples. In these cases, simply fitting and extrapolating the pre-edge background with the function above can yield inconsistent and unexpected results [2]. While many of these issues can be addressed [3-5], the established tools are often inadequate to deal with the noisy spectra with limited available pre-edge regions that are common when mapping elements or electronic structure in STEMEELS. In this study, we present a multi-region background fitting method targeted at overcoming such problems.

Just as the background under a given core-loss EEL edge follows a power law, the post-edge decay obeys the same functional form [1]. This structure can be used to constrain and refine the background fitting function by simultaneously fitting the pre-edge background and post-edge decay of a core-loss edge with:

$$
\begin{aligned}
& I_{\text {pre }}(E)=A_{1} E^{-B_{1}}, \text { and } \\
& I_{\text {post }}(E)=A_{1} E^{-B_{1}}+A_{2} E^{-B_{2}},
\end{aligned}
$$

where $I_{\text {pre }}$ is fit to a $10-30 \mathrm{eV}$ region directly prior to the edge onset, and $I_{\text {post }}$ is fit to a similarly-sized region past any extended-loss features of the edge. Constraints are used on $A_{i}$ and $B_{i}$ to maintain proper power law behavior of the fit. Due to the simultaneous fitting and shared variables between each region, the post-edge fit penalizes deviations from power-law behavior in the post-edge decay, thereby refining the pre-edge fit. This results in a far more reliable background fit even when faced with exceptionally noisy data with limited fitting regions, such as the example in Figure 1, enabling more consistent direct comparison and quantification of core-loss edge structures.

By following the same pattern for additional regions, this approach can be extended to fit multiple edges in the same spectrum simultaneously, producing multiple background-subtracted EEL edges, as shown in Figure 2. With this method, each subsequent fitted region refines the rest, producing a consistent and robust removal of background signal from each core-loss EEL edge in the spectrum [6,7]. 
References:

[1] RF Edgerton in "Electron Energy-Loss Spectroscopy in the Electron Microscope", $3{ }^{\text {rd }}$ edition. (Springer).

[2] D-R. Liu et al., J. Microsc. 147 (1987), p. 37.

[3] H Tenailleau et al., J. Microsc. 166 (1992), p. 297.

[4] J Verbeeck et al., Ultramicroscopy 101 (2004), p. 207.

[5] G Bertoni, et al., Ultramicroscopy 108 (2008), p. 782.

[7] This work was supported primarily by NSF through the University of Minnesota MRSEC under Award Number DMR-1420013.
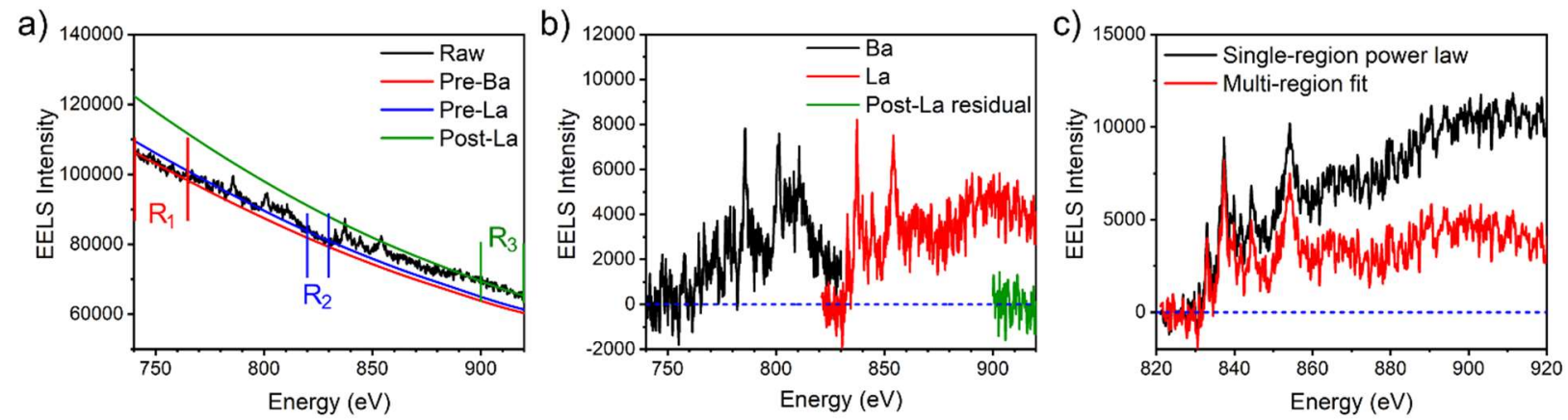

Figure 1. Background subtraction from a noisy EEL spectrum obtained near a La-doped BSO/SSO interface where the La spectrum was of particular interest. (a) Raw spectrum with fitted regions $\mathrm{R}_{1-3}$ indicated along with corresponding fit backgrounds using a 3-region fit following the pattern in Eqns. 1 and 2. (b) Background-subtracted Ba and La M edges. c) La M edge with the background removed via a single-region power law fit compared with this multi-region method.
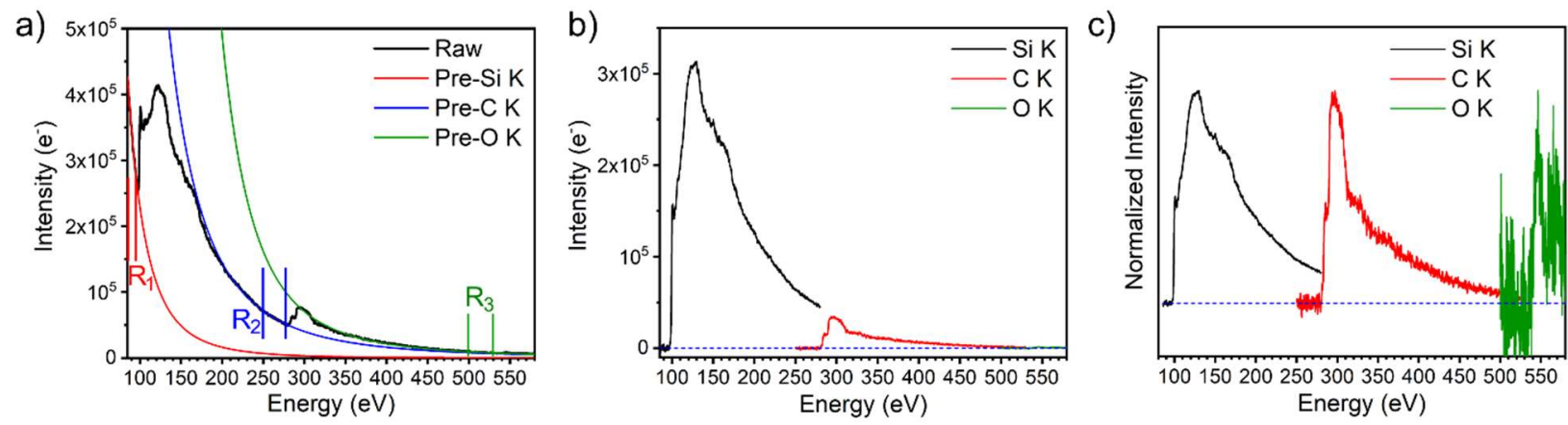

Figure 2. Multi-region background subtraction applied to an EELS spectrum collected at the center of a slightly oxidized $8 \mathrm{~nm} \mathrm{Si} \mathrm{nanocrystal} \mathrm{sitting} \mathrm{on} \mathrm{a} \mathrm{thin} \mathrm{carbon} \mathrm{grid,} \mathrm{demonstrating} \mathrm{the} \mathrm{simultaneous}$ removal of background signal from multiple edges. (a) Raw spectrum with fitted regions $\mathrm{R}_{1-3}$ indicated along with corresponding background fit curves. (b) $\mathrm{Si}, \mathrm{C}$, and $\mathrm{O} \mathrm{K}$ edges after background subtraction. (c) The background-subtracted edges from (b) rescaled to be simultaneously visible, showing healthy and consistent background subtraction for each edge. 